\title{
Uma proposta de incorporação dos estudos sobre inovação nas pesquisas em jornalismo ${ }^{1}$
}

\section{Carlos Eduardo Franciscato*}

Artigo recebido em: 15 de abril de 2010 Aprovado em: 9 de maio de 2010

* Jornalista, professor da

Universidade Federal de Sergipe. Doutor em Comunicação e Cultura Contemporâneas pela Universidade Federal da Bahia. Atualmente é presidente da Associação Brasileira de Pesquisadores em Jornalismo (SBPJor).

cfranciscato@uol.com.br

Resumo: O termo 'inovação' tem uso corrente no setor produtivo, por ser um indicador de um movimento de geração de conhecimento vinculado à produção. Da mesma forma, agências de fomento à pesquisa apoiam projetos que articulem a ciência ao desenvolvimento de conhecimento aplicado ao ambiente produtivo. Embora na raiz da ideia de inovação exista uma identidade com a investigação, a descoberta e o crescimento do saber à semelhança da academia, os estudos em jornalismo escassamente incorporam esta perspectiva aplicada do conhecimento científico na forma de uma terminologia e conceituação adequadas ao avanço dos estudos sobre a atividade jornalística. O objetivo deste artigo é investigar as razões deste distanciamento, mesmo sendo o jornalismo uma atividade social que poderia se beneficiar diretamente da aplicação do conhecimento científico. Procuraremos também desenvolver o termo 'inovação' para além da formulação conceitual frágil que tem caracterizada sua utilização. Indicaremos que, no jornalismo, uma dimensão mais complexa da ideia de inovação exige considerar três vertentes deste fenômeno (inovação tecnológica, organizacional e social), para, assim, possibilitar uma maior densidade conceitual e superar um viés excessivamente tecnológico em suas abordagens.

Palavras-chave: Teorias do jornalismo; pesquisa aplicada; inovação.

\section{A proposal of incorporation of the studies on innovation in journalism researches}

\begin{abstract}
The term 'innovation' is commonly used in the productive sector because it indicates a movement of knowledge generation linked to the production. In the same way, agencies of research promotion support projects that connect science with the development of knowledge applied to the productive environment. Although the idea of innovation brings in its core an identity with the investigation, the discovery and the know growth that is found in the academy, journalism studies barely incorporate this applied perspective of the scientific knowledge shaped in a terminology and conception that is appropriate to the advance of the studies about the journalistic activity. This article aims to investigate the reasons for this estrangement, although the journalism is a social activity that could directly benefit from the application of the scientific knowledge. We will also pursue to develop the term 'innovation' by going beyond its fragile conceptual formulation that has characterized its use. We will indicate that, in journalism, a more complex dimension of the idea of innovation demands the consideration of three aspects - technological, organizational and social innovation. That way we will make it possible to achieve a greater conceptual denseness in order to surpass an excessively technological bias in its approaches.
\end{abstract}

Keywords: Theories of journalism; applied research; inovation.

\footnotetext{
'Este artigo foi produzido com resultados da pesquisa "Desenvolvendo modelos de Pesquisa Aplicada em Jornalismo - Dois estudos de caso", projeto financiado pelo CNPq no Edital 50/2006, tendo a participação dos bolsistas Getúlio Cajé dos Santos (Apoio Técnico) e Dijna Andrade Torres (PIBIC).
} 
literatura que vem estudando as interações entre universidades e institutos de pesquisas e organizações do setor produtivo tem indicado a necessidade de um duplo comprometimento: de um lado, as empresas devem desenvolver competências tecnológicas que possibilitem a absorção efetiva de conhecimento gerado nas universidades. Do lado da academia, o conhecimento científico deve assumir forma e conteúdo de assistência direta para as necessidades de mudança dos vários setores industriais (Rapini e Righi, 2006, p. 134).

As contribuições que as universidades podem oferecer para o processo inovativo nas empresas são sintetizadas por Rapini e Righi (2006) como: a) fonte de conhecimento de caráter mais geral necessários para as atividades de pesquisa básica; b) fonte de conhecimento especializado relacionado à área tecnológica da firma; c) formação e treinamento de engenheiros e cientistas capazes de lidar com problemas associados ao processo inovativo nas firmas; d) criação de novos instrumentos e de técnicas científicas; e e) criação de firmas nascentes (spin-offs) por pessoal acadêmico.

Ao mesmo tempo, Rapini (2004) identificou as dificuldades na interação universidade-empresa para constituição de parceria que envolvam desenvolvimento ou troca de conhecimentos: a) baixo conteúdo científico e curto prazo requerido para as soluções industriais que não estimula os contratantes a investirem em C\&T; b) ausência de interlocutores adequados nas empresas dificultando a comunicação; c) setor produtivo pouco inovativo; d) ausência de instrumentos adequados nas universidades para a comercialização de tecnologia; e e) pouca flexibilidade das instituições de C\&T.

Este contexto de desafio na interação entre academia e setor produtivo (não somente o setor privado de perfil industrial e visando ao lucro, mas todas as empresas, organizações e coletividades estruturadas em torno de unidades produtivas de jornalismo) serve, em linhas gerais, para compreender as relações entre universidades e empresas de comunicação no Brasil, particularmente as jornalísticas. As iniciativas são isoladas, e o cenário comum é o de dois atores sociais caminhando paralelamente, com escassos pontos de contato. Ao mesmo tempo, ambos têm na geração de conhecimento o alicerce para suas atividades, seja ele na forma predominantemente conceitual (academia) ou aplicada à produção (organizações jornalísticas).

No setor produtivo, a geração de conhecimento é mais frequentemente definida com o termo "inovação". Embora na raiz do termo exista uma identidade com a investigação, a descoberta e o crescimento do saber à semelhança da academia, os estudos em jornalismo pouco incorporam esta perspectiva aplicada do conhecimento científico na forma de uma terminologia e conceituação adequadas ao avanço dos estudos sobre a atividade jornalística.

O objetivo deste artigo é investigar as razões deste distanciamento, mesmo sendo o jornalismo uma atividade social que poderia se beneficiar diretamente da aplicação do conhecimento científico. Procuraremos também desenvolver o termo 'inovação' para além da formulação conceitual frágil que tem caracterizada sua utilização. Indicaremos que, no jornalismo, uma dimensão mais complexa da ideia de inovação exige considerar três vertentes deste fenômeno (inovação tecnológica, organizacional e social), para, assim, possibilitar uma maior densidade conceitual e superar um viés excessivamente tecnológico em suas abordagens. 


\section{Tipos de conhecimento no campo do jornalismo}

Para os propósitos desta investigação, recorremos ao conceito de reflexividade, central na teoria social de Giddens. Para o autor, a vida social consiste no fato de que as práticas sociais são constantemente examinadas e reformadas a partir da informação renovada sobre estas próprias práticas, alterando assim constitutivamente seu caráter (1991, p. 45). A reflexividade do conhecimento se dará por este ocorrer em um processo de mútua influência: o conhecimento se realiza não apenas como um discurso sobre o mundo social, mas contribui para constituir este mundo, ao mesmo tempo em que a experiência social altera o conhecimento. "O conhecimento novo (conceitos, teorias, descobertas) não torna simplesmente o mundo social mais transparente, mas altera sua natureza, projetando-a para novas direções” (Giddens, 1991, p. 153).

A partir de uma percepção do caráter reflexivo e circular do conhecimento na sociedade, consideramos três competências específicas que geram conhecimentos como relevantes para a constituição do campo do jornalismo (Bourdieu, 1997). A primeira competência é aquela geradora de conhecimento científico. Este conhecimento é resultante de um esforço histórico de formulação nos estudos sobre jornalismo, independente das sub-áreas do conhecimento a que estes estudos se filiem.

A segunda competência pode ser nomeada como 'conhecimento especializado': é aquele produzido por atores com domínio e saber técnico em uma área específica, gerando um saber complexo, envolto em técnicas, práticas, procedimentos e regramentos, mas não necessariamente científico. Tal modo pode ser mais bem compreendido a partir da conceituação de Giddens de "sistemas peritos", os quais, para o autor, são "sistemas de excelência técnica ou competência profissional que organizam grandes áreas dos ambientes material e social em que vivemos" (1991, p. 35). Estes sistemas são reconhecidos socialmente por meio de um tipo de "fé" que depositamos sobre a competência daqueles que detêm o "conhecimento perito".

O jornalismo é um sistema perito porque confiamos na competência técnica do jornalista e em seu compromisso de procurar relatar fatos socialmente relevantes de forma equilibrada, com a exatidão possível e com intenção de fidelidade ao real. Não temos como comprovar se, a cada momento, o jornalista está executando isto, mas, no conjunto, confiamos no aparato técnico da profissão. $\mathrm{O}$ jornalismo opera com três níveis de saberes especializados que conformam a atividade e o conteúdo jornalístico: a) os saberes ativados cotidianamente pela comunidade profissional ao realizarem suas práticas jornalísticas; b) o setor produtivo, que detém o controle estrutural e organizacional do processo de produção jornalística; e c) as fontes de informação ouvidas pelos repórteres, cujo conhecimento especializado sobre temas e situações torna-as autorizadas a contribuir com o jornalista com informações e interpretações para a construção do relato sobre o fato.

A terceira competência manifesta-se na produção de um conhecimento leigo, resultante de noções não sistematizadas nem operacionalizadas sobre o jornalismo. Este é o conhecimento que o público tem sobre o jornalismo: não domina suas técnicas, seus procedimentos, nem seus conceitos, mas tem uma noção elementar do que é o jornalismo e do papel dele em suas vidas. Neste 
conjunto de saberes situam-se tanto a crença do público na funcionalidade do sistema perito do jornalismo quanto nos valores, curiosidades, interesses e expectativas que, ao serem captadas pela organização jornalística, são, em alguma medida, incorporadas pelos jornalistas na sua prática e contribuem para a conformação do produto noticioso.

O conhecimento leigo do público sobre o jornalismo completa o ciclo de conhecimentos que constituem o campo do jornalismo, só que de uma forma reativa. Os conhecimentos que os atores do campo do jornalismo produzem em suas práticas (sejam conceitos, procedimentos e conteúdos jornalísticos) sofrem um caráter reflexivo nos processos de recepção social das notícias ou dos discursos expressos sobre elas. A circulação das notícias ocorre com base em valores e qualidades legitimados socialmente e reforçados a cada novo ciclo de circulação, os quais se manifestam em procedimentos práticos (reações dos leitores em contato com as redações dos jornais) ou especializados (pesquisas de audiência).

\section{O conceito de inovação e sua aplicabilidade no jornalismo}

Desenvolver uma geração compartilhada de conhecimentos entre academia e setor produtivo pressupõe o enfrentamento de alguns problemas que são gerais à interação entre os dois atores sociais, mas com graus diferenciados em áreas diversas. Ao conduzirmos esta discussão para o campo do jornalismo, pretendemos localizar especificidades nesta interação. Inicialmente, concordamos com Meditsch (2004, p. 99) ao considerar que a indústria jornalística brasileira, mesmo que busque nas universidades para a solução de seus problemas, tem dificuldades em encontrar interlocutor interessado ou capacitado para esta parceria.

Pretendemos demonstrar os desafios entre academia e empresas jornalísticas a partir de uma análise e investigação teórica a respeito do conceito de inovação, em três aspectos: inovação tecnológica, organizacional e social. Acreditamos que este conceito, estratégico para as noções de pesquisa e desenvolvimento nas empresas, tem encontrado fecundidade acadêmica também em áreas do conhecimento como economia, sociologia e engenharia. Mais do que mostrar distanciamentos, nosso esforço será de buscar o que Rapini e Righi identificam como "avanços no desenvolvimento de uma linguagem em comum entre os grupos de pesquisa e o setor produtivo" (2006, p. 152-153).

Iremos investigar as possibilidades de formulação desta "linguagem em comum" por meio de uma tentativa de compreensão e inserção do conceito de inovação no campo do jornalismo, à luz das possibilidades de produção de conhecimento acadêmico e aplicado neste campo. Primeiro, é necessário buscar em Moreira e Queiroz (2007, p. 6-9) uma primeira abordagem do conceito, referindo-o a noções mais amplas como "invenção" ou "adoção" de processos ou produtos novos para uma organização ou para um ambiente em que determinada atividade é realizada. As teorias econômicas e da administração fizeram uma apropriação e elaboração do conceito, priorizando sua aplicação a questões sobre desenvolvimento tecnológico, econômico e empresarial.

Recorreremos a estas formulações na medida em que elas forem necessárias 
para localizar pontos de contato na terminologia, definição, interesses e usos próximos de conhecimentos sobre o jornalismo tanto da academia quanto do setor produtivo. Isto pode ser mais bem realizado considerando as três dimensões da inovação (tecnológica, organizacional e social).

\section{Inovação tecnológica}

A inovação tecnológica indica, pelo termo, uma vinculação a procedimentos que envolvem geração ou aplicação de tecnologias no jornalismo. O desenvolvimento tecnológico por que tem passado as indústrias da mídia e, particularmente, o jornalismo, tem se acentuado nos últimos anos, tanto pelo processo de digitalização das ferramentas e conteúdos quanto pela conexão e disponibilização de produtos por redes telemáticas.

O que é necessário considerar em uma tentativa de compreensão das formas de conhecimento produzidas no campo do jornalismo levando-se em conta o eixo conceitual da inovação é observar que a tecnologia não é mera ferramenta de produtividade em um sentido mecânico de aceleração dos processos de trabalho, mas sim observar, com Castells (2001), uma complexa interação entre tecnologia e sociedade, particularmente no campo da produção e circulação de informação. Mas Castells acrescenta a este binômio o conhecimento, em um "círculo virtuoso de interação entre as fontes de conhecimentos tecnológicos e a aplicação da tecnologia para melhorar a geração de conhecimentos e o processamento da informação" (2001, p. 35).

A inovação tecnológica no jornalismo, portanto, não pode ser considerada como um investimento isolado em modernização industrial, mas caracterizada também como um aporte que modifica as rotinas e processos de trabalho do jornalista, bem como o perfil e a qualidade do produto jornalístico. Um produto tecnologicamente novo, conforme a Pesquisa de Inovação Tecnológica 2005 realizada pelo IBGE, é aquele cujas características fundamentais (especificações técnicas, componentes e materiais, software incorporado, funções ou usos pretendidos) diferem significativamente de todos os produtos previamente produzidos. Já a inovação tecnológica de processo refere-se à introdução de tecnologia de produção nova ou significativamente aperfeiçoada, assim como de métodos novos ou substancialmente aprimorados de oferta de serviços ou para manuseio e entrega de produtos (Pintec, 2005, p. 19-20).

Partindo da discussão sobre a relação entre tecnologia e sociedade, a direção tomada por Guilherme Plonski (2005) para definir inovação tecnológica é de que esta pode ser entendida como um fenômeno marcadamente socioeconômico, que envolve mudanças e empreendedorismo. Ou seja, de acordo com o autor, a inovação tecnológica vai além do conceito que afirma que esse tipo de inovação se resume somente a ações técnicas e produtos tecnologicamente novos utilizados dentro de grandes empresas para suprir necessidades de determinada parcela da sociedade.

Para Plonski (2005), a inovação tecnológica é vista como uma estratégia para auxiliar empresas, regiões e países, solucionando problemas e aflições, para que seja promovido seu desenvolvimento. $\mathrm{O}$ autor afirma que, como qualquer tipo de inovação, a inovação tecnológica envolve mudança. Porém, não envol- 
ve somente mudanças de base técnica e de caráter incremental. As inovações tecnológicas não são apenas produtos e processos criados com intenção de posicionar empresas no mercado, elas também são ações de execução dentro de empresas que favoreçam ou beneficiem a sociedade.

Para Bastos Tigre (2006), as empresas inovadoras recorrem a fontes internas e externas de conhecimento. As fontes internas são relativas às atividades voltadas para o desenvolvimento de produtos, processos e programas para melhoria da empresa. As fontes externas são aquelas que envolvem a aquisição de produtos, tecnologias, consultorias de terceiros, para qualificar os funcionários e a estrutura da empresa. As empresas selecionam as fontes que sejam mais adequadas, ou seja, que estejam associadas às estratégias adotadas pelas empresas e características do tipo de tecnologia que as organizações necessitam.

Exemplos de inovações tecnológicas nos jornais impressos podem ser localizados em várias etapas do processo de produção, atingindo também a impressão. Nas redações, um dos primeiros processos a introduzir, de forma sistemática, a plataforma digital foi a substituição das máquinas de escrever e computadores na década de 1980 em várias empresas brasileiras (Silva, 1988; Baldessar, 2003; Fonseca, 2005), processos que ocorreram concomitantes a mudanças na gestão organizacional, administrativa e no lançamento de novos produtos jornalísticos.

Isto significa que a inovação em uma empresa jornalística não ocorre isoladamente, mas dentro de uma cadeia de ações e efeitos. Um processo mais recente de inovação tecnológica, ainda em suas etapas iniciais, é a convergência de mídias, que indica uma transformação profunda na estrutura, ambiente e rotina de trabalho das redações (Quinn, 2005). As empresas brasileiras já estão estudando formas de se preparar para estas transformações, que atingem também as funções dos jornalistas dentro da empresa e a indicação de novos conhecimen-

A inovação em uma empresa jornalística não ocorre isoladamente, mas dentro de uma cadeia de ações e efeitos tos como requisitos para a permanência no mercado, como o jornalista "multifuncional".

\section{Inovação organizacional}

Consideramos esta um segundo grau de inovação nas empresas jornalísticas. Conforme o Manual de Oslo, documento desenvolvido pela Organização para Cooperação Econômica e Desenvolvimento (OCDE) e publicado no Brasil pela Financiadora de Estudos e Projetos (FINEP) como forma de estimular o desenvolvimento da inovação nas empresas, uma inovação organizacional é a "implementação de um novo método organizacional nas práticas de negócios da empresa, na organização do seu local de trabalho ou em suas relações externas” (Manual de Oslo, 2000, p. 36).

A inovação organizacional é, segundo este estudo, um importante passo para o desenvolvimento das empresas, e passa também por melhorar o nível de aprendizado e de conhecimento dos atores envolvidos na produção, o que poderia ser viabilizado com programas de treinamento e capacitação 
ou cursos de aperfeiçoamento profissional. Barañano (2005, p. 68) considera que a gestão das empresas inovadoras deve ser suportada por uma estrutura organizacional que torne viável o esforço de inovação, caso contrário esta estrutura será uma barreira ou, pelo menos, atrasará qualquer iniciativa inovadora. Conforme Moreira e Queiroz, a diferença entre inovação tecnológica e inovação organizacional é de que esta última possui um caráter interativo: "As inovações na estrutura organizacional incluem mudanças nas relações de autoridade, nas alocações de trabalho, nos sistemas de remuneração, nos sistemas de comunicação e em outros aspectos da interação formal entre as pessoas na organização" (Moreira e Queiroz, 2006, p. 9-10).

As inovações organizacionais são mudanças nas rotinas de trabalho, com implantação de novos modelos de gestão, novos ambientes e as formas como inovações tecnológicas podem induzir à criação de novos processos. No jornalismo, as inovações organizacionais englobariam deste o trabalho de apuração do repórter até os procedimentos de edição e finalização técnico-industrial do produto. Talvez o exemplo mais clássico destas interrelações entre tecnologia e organização (e das particularidades de cada uma) foi descrito por Lins da Silva (1988) ao analisar o projeto de transformação editorial e empresarial do jornal Folha de S. Paulo na década de 1980.

\section{Inovação social}

Além dos aspectos tecnológico e organizacional, avaliamos que as empresas jornalísticas alcançam um terceiro tipo de atitude inovativa, que alguns autores denominam de inovação social. Trata-se de um termo que indica os usos ou efeitos sociais dos processos interativos, bem como as interações que agentes sociais desenvolvem com as organizações jornalísticas. André e Abreu (2006, p. 124) consideram que a inovação social possui três atributos: satisfação de necessidades humanas não satisfeitas por via do mercado; promoção da inclusão social; e capacitação de agentes ou atores sujeitos a processos de exclusão/ marginalização social. Segundo os autores,

A inovação tecnológica foca-se no objecto e as primeiras concepções da inovação social incidem sobre o contexto (emprego, qualificação, segurança social, território) - uma focagem diferente, intenções convergentes. As perspectivas mais recentes afastam definitivamente a inovação social da tecnológica, atribuindo-lhe uma natureza não mercantil, um carácter colectivo e uma intenção que não só gera, mas também visa, transformações das relações sociais (André e Abreu, 2006, p. 125).

A inovação social está ligada à ideia de desenvolvimento social, em uma perspectiva que amplia um foco mercadológico. Moreira e Queiroz (2001) consideram haver uma interação entre novas ideias, produtos e práticas, função social e estrutura. Para os autores, as inovações podem criar mudança social, e a subsequente mudança social trazer inovações adicionais que podem 
reagir sobre as estruturas e/ou funções alteradas que as fizeram existir ou influenciar outros aspectos da organização.

Plonski (2005) considera que o impacto de uma inovação social na sociedade pode ser muito maior do que o produzido por uma inovação tecnológica ou organizacional. Como exemplo de inovação social Plonski cita o jornal. Ao mesmo tempo, uma análise mais detida da história do jornalismo no século XIX mostra que ele foi, na verdade, o desenvolvimento combinado de sucessivas inovações tecnológicas, organizacionais e, também, sociais.

Este terceiro processo inovativo, a inovação social, permite identificar de que maneira fatores tecnológicos que vêm transformando os modos de fazer jornalismo, como a digitalização dos processos e produtos jornalísticos e a presença e formas de participação das organizações jornalísticas em redes de base tecnológica (com outras empresas ou em relação aos seus públicos) que possibilitem ganhos operacionais e tecnológicos, bem como novas formas de interação com a sociedade e inovações na atividade jornalística.

A inovação tecnológica cria condições para que seja produzido: a) um produto jornalístico de melhor qualidade, b) um benefício social da aplicação desta tecnologia ao jornalismo; e c) um uso social deste produto ou uma maior interação entre a sociedade e as organizações jornalísticas. De forma ilustrativa podemos citar a infografia no jornalismo, um novo produto jornalístico, tecnologicamente estruturado, que articula criativamente linguagens e conteúdos sobre uma lógica visual (para os veículos impressos) ou audiovisual (para as demais mídias, incluindo a Internet).

\section{Produção de conhecimentos na interação academia-setor produtivo}

As três noções de inovação serão, agora, colocadas em diálogo com formas e processos de construção científica do conhecimento no jornalismo. Assim, por meio deste esforço, pretendemos identificar aproximações, possibilidades e desafios na interação entre a academia e o setor produtivo.

As organizações jornalísticas ocupam, no campo do jornalismo, uma posição de controle sobre a estrutura produtiva, focalizando suas estratégias de geração de conhecimento para o objetivo de garantir sua reprodutibilidade, preservação e ampliação de espaços na sociedade e mercado e, nas empresas privadas, obtenção de lucro e acumulação de capital. Esta racionalidade sistêmica faz estas organizações privilegiarem uma lógica de produção de conhecimento voltada para a execução eficiente de atividades e obtenção de resultados.

É compreensível, portanto, que as noções de inovação apresentadas anteriormente sejam as que representem, para os profissionais que gerenciam esta estrutura, as formas de conhecimento valorizadas. A própria capacidade lógica de formulação de conhecimento está condicionada pelas tensões de operação e resultados. Isto significa a construção de uma terminologia, linguagem e definições de apoio às suas atividades e a secundarização ou distanciamento de outras formas.

Diferentemente do setor produtivo, a academia tem na produção de conhe- 
cimento sua atividade fim, principalmente o conhecimento científico. Mais do que isto, a academia desenvolve métodos para construir e validar este conhecimento, a fim de tornar este discurso uma verdade à luz da ciência. Algumas práticas sistêmicas e mesmo mercadológicas podem permear certos processos institucionais da ciência, mas não sua razão de ser.

O conceito de inovação não tem sido objeto de estudo no campo da comunicação nem nos estudos em jornalismo, à exceção do livro organizado por Caprino (2008). Por a origem do conceito estar articulada ao ambiente e à lógica da produção, seus referenciais teóricos vinculam-se também ao campo das Ciências Sociais Aplicadas (economia e administração principalmente) e não ao das Ciências Humanas. Isto significa que um projeto estratégico que os pesquisadores possam executar para possibilitar a interação academia-empresa no campo do jornalismo necessita construir uma linguagem e uma terminologia afins entre os atores e, no caso da academia, incorporar e manusear termos e definições do campo das Ciências Sociais Aplicadas.

Isto gera, conforme Sassen (2002), um desafio adicional à comunidade científica, particularmente aqueles pesquisadores do campo das ciências humanas e sociais aplicadas, que possuem uma autoridade socialmente constituída para formular, sistematizar, descrever e analisar processos sociais. O que significa

A academia tem

na produção de conhecimento sua atividade fim, principalmente o conhecimento científico

um reforço ao item anterior sobre o papel da academia na construção da interação com o setor produtivo:

The challenge for sociology is not so much to deny the weight of technology, but rather to develop analytic categories that allow us to capture the complex imbrications of technology and society (Sassen, 2002, p. 365).

\section{Considerações finais}

Ao desenvolvermos uma discussão teórica sobre as possibilidades de geração do conhecimento no campo do jornalismo em condições de interação entre academia e setor produtivo e utilizar o conceito de inovação como objeto de análise, procuramos executar um percurso que, primeiro, indicasse uma das raízes deste distanciamento, de ordem epistemológica, sobre a natureza, valor e uso dos conhecimentos produzidos por cada ator. Tal distanciamento está articulado a uma segunda dimensão, que se refere às metodologias de produção deste conhecimento.

A apresentação das três noções de inovação (tecnológica, organizacional e social) teve a intenção de possibilitar a incorporação de uma abordagem analítica ao estudo. Ressaltamos que, para além das transformações de ordem tecnológica e organizacional que as organizações jornalísticas vêm passando nas últimas décadas, consideramos oportuna a noção de inovação social para visualizar o aspecto imbricado da tecnologia e seu uso social.

As instituições jornalísticas, ao alimentarem cotidianamente a sociedade com um fluxo noticioso sobre o mundo, constroem, reforçam e modificam processos sociais e culturais. Para além de sua dimensão mercadológica, cada produto noticioso apresentado à sociedade traz, mesmo que implícitos, estes 
elementos socioculturais, sedimenta ou altera hábitos, conhecimentos e valores sociais a curto ou a longo prazos.

O conceito de inovação social impede, também, os riscos de que a intensiva transformação tecnológica da sociedade revigore perspectivas como a de "difusão de inovações" na sua intenção de criar modelos de disseminação de novas tecnologias para a sociedade. Ao identificarmos a complexidade do social inerente à presença de atores múltiplos (não só a academia e o setor produtivo, mas redes sociais colaborativas no desenvolvimento de tecnologias, processos e produtos), procuramos sinalizar que a geração de conhecimentos no campo do jornalismo, com seus desafios de melhoria na qualidade, produtividade e uso social, pressupõe uma construção compartilhada deste processo entre atores institucionais e não-institucionais.

\section{Referências bibliográficas}

ANDRÉ, Isabel; ABREU, Alexandre. Dimensões e espaços da inovação social. Disponível em Internet: http://www.ceg.ul.pt/finisterra/numeros/200681/81_06.pdf. Capturado em 05/02/08.

BARÃNANO. Ana María. Gestão da Inovação Tecnológica: Estudo de Cinco PMEs Portuguesas. Revista Brasileira de Inovação. Vol 4, No 1 Jan/Jun 2005, p. 57 a 96.

BOURDIEU, Pierre. Sobre a Televisão. Rio de Janeiro: Jorge Zahar, 1997. CAPRINO, Mônica (org.). Comunicação e Inovação. São Paulo: Paulus, 2008.

CASTELLS, Manuel. A Sociedade em rede - A era da informação: economia, sociedade e cultura (vol. 1). São Paulo: Paz e Terra, 2001.

FONSECA, Virginia P. S. O Jornalismo no Conglomerado de Mídia - Reestruturação produtiva sob o capitalismo global. Tese de Doutorado apresentada ao Programa de Pós-Graduação em Comunicação e Informação da UFRGS. Porto Alegre (RS), 2005.

GIDDENS, Anthony. As consequências da modernidade. São Paulo: Editora UNESP, 1991.

MANUAL DE OSLO - Diretrizes para Coleta e Interpretação de Dados Sobre Inovação. Organização para Cooperação e Desenvolvimento Econômico / Gabinete Estatístico das Comunidades Europeias, 2000. Disponível em Internet: http://www.finep.gov.br/dcom/brasil_inovador/capa.html. Capturado em 20/05/07.

MEDITSCH. Eduardo. Estudos em Jornalismo. Revista Brasileira de Ciências da Comunicação. Vol XXVII, n 2, jul/dez 2004. São Paulo: INTERCOM, p. 93-107.

MOREIRA, Daniel; QUEIROZ, Ana Carolina. Inovação: conceitos fundamentais. In: Thomson Learning, 2006, p. 01-22.

PAVLIK, John. Journalism and new media. New York: Columbia University Press, 2001.

PESQUISA DE INOVAÇÃO TECNOLÓGICA - PINTEC2005. Instituto Brasileiro de Geografia e Estatística - IBGE. Rio de Janeiro, 2007. 
QUINN, Stephen. Convergent Journalism: The Fundamentals of Multimedia Reporting. New York: Peter Lang Publishing, 2005.

RAPINI, M.S., Interação Universidade-Indústria no Brasil: Uma análise exploratória a partir do Diretório de Pesquisas do CNPq. Dissertação de Mestrado apresentada ao Instituto de Economia da UFRJ, Rio de Janeiro, 2004.

RAPINI. M; RIGHI, Hérica. O Diretório dos Grupos de Pesquisa do CNPq e a Interação Universidade-Empresa no Brasil em 2004. Revista Brasileira de Inovação. Vol 5, No 1, Jan/Jun 2006.

SASSEN, Saskia. Towards a Sociology of Information Technology. Current Sociology, Vol 50 (3), May 2002, p. 365-388 